\title{
EQUAL:Towards an Inclusive Design Approach to Novice Programming Languages and Computing Environments for Native Users
}

\author{
Basawaraj Patil, Klaus Maetzel and Erich. J. Neuhold \\ Fraunhofer Institute for Integrated Publication and Information Systems, Dolivostrasse 15, \\ 64293, Darmstadt, Germany \{patil,maetzel,neuhold\}@ipsi.fraunhofer.de
}

\begin{abstract}
In the current textual programming languages (conventional, novice, etc.) and programming paradigms (e.g., procedural, declarative, functional etc.), the programming constructs, semantic concepts, and syntactic elements are based on English paradigm and implemented using ASCII character sets, seriously limiting the universal access to programming and computing skills. Especially, non-English speaking native users (students, adults etc.) from non-English speaking geographical regions, including visually challenged users, encounter serious cognitive, semantic and syntactic difficulties in understanding and translating their programming plans into the syntax and semantics of English based paradigm of a programming language. Authors have developed an inclusive, universal design framework with flexible cognitive, semantic and syntactic, and cultural adaptations in the textual languages and their compilers/interpreters to satisfy the computing requirements of native users.
\end{abstract}

Key words: native users, novice programming, universal access, universal design, textual languages, and universal usability.

\section{INTRODUCTION}

In the evolving Information Society, programming, computing and IT skills are becoming important in educational, professional and personal endeavours. With globalisation and rapid developments in IT, the majority of Internet users do not use English. Native users' issues may become serious because the vast majority of the world's population who do not, and 
will not in the foreseeable future speak English will be excluded from the Information Society. Students and people lacking programming and computing skills may become a serious individual and social problem (Dyson, 1997) and in the worst case, may lead to - Internet Apartheid (Shneiderman, 2000). Native users referred to here include users, such as students (e.g., primary and high schools, colleges and universities), adults, indigenous societies, linguistic minorities, including people with special needs, from non-English speaking geographical regions (e.g., Asia, the Middle East, Europe, Africa and South America). In particular, millions of native students, for perhaps political, social, economical reasons are compelled to learn in their native medium, and have no learning opportunities to acquire programming skills. We use the generic term textual languages to cover a broad spectrum of textual information structures, command and macros, query and programming languages that inherently textual in nature. Lack of appropriate technologies that support Universal Access (UA) to linguistic and cultural content, textual languages and vernacular computing resources have been a serious impediment to the development of computer literacy skills and programming expertise. Thus, Universal Access to textual languages and programming languages (e.g., novice, conventional), constitute a major step towards an Inclusive Society and helps in reducing Digital Divide gap.

In teaching and learning of programming languages and computing skills with English as programming paradigm will seriously limit universal access to programming and computer literacy skills. Because of historical reasons, the majority of novice programming languages, such as LOGO, BASIC, conventional programming languages such as $\mathrm{C}, \mathrm{C}++$, Java and programming paradigms, declarative, procedural, functional, object oriented are mainly based on English semantics and syntax and implemented using ASCII character sets. Native students and adults, whose medium of education and instruction is in their native medium and socio-cultural environments, encounter enormous linguistic, cognitive, semantic, syntactic, and cultural difficulties in understanding and translating their programming plans into English-based semantics and syntax of a programming language. In the new technology adaptation for Educational Computing and Learning, non-availability and non-accessibility of programming languages and computing environments and language processors (e.g., interpreters, compilers, translators) constitute major stumbling block to harness the power of IT technologies (Rogers, 2000).

In this paper, we focus on our research work related to the universal usability issues of textual languages as found in programming languages, language processors and, computing environments from native users' perspectives with more emphasis on semantics and syntax i.e., notational 
aspects of programming languages and programming constructs. We also discuss a universal, inclusive design methodology of textual information structures and programming languages, language processors and computing environments. Furthermore, it will contribute to the better understanding of native user requirements, universal design of programming languages and computing environments, and implications to scaffolding and teaching tools.

\section{NATIVE USERS: A PROFILE AND THEIR COMPUTING REQUIREMENTS}

Research studies on needs and computing requirements of native users are scarce and poorly understood. In-house studies on universal usability textual languages, novice programming languages and programming paradigms were conducted with the help of researchers, $\mathrm{HCI}$ experts, native users and students with diverse multilingual and multicultural backgrounds. From these preliminary studies, we may characterise computing requirements of native users as follows:

- A majority of native users are novices and lack English knowledge. They prefer native language-like constructs and interactions in programming and computing tasks and vernacular computing environments. They want to learn basic skills of programming and computing and do not intending to become professional programmers. These native users need enabling mechanisms, processes and technologies to enable them to acquire the basic skills of programming and computing.

- A majority of native users, especially students with good mathematical background would like learn basic and advanced programming skills, and intend to become professional programmers. These native users need special scaffolding mechanisms, processes and technologies to make a effective transition from native computing to the main stream of computing.

- A majority of native users have special needs. For example, native visually challenged users prefer to interact and program in native Braille (e.g., Asian, European, and African Braille) or native voice interactions. These users need assistive mechanisms, processes and technologies to avail new opportunities, facilities and services to enhance their quality of life. 


\section{USER HCI MODEL AND PROGRAMMING LANGUAGE ISSUES}

Programming is a complex activity and programming languages make the task more difficult than necessary because, the programming languages are designed without serious considerations of human factors and $\mathrm{HCI}$ issues (Pane et al., 2001). In general, programming is the task of mapping the mental plans and program compositions into language constructs, i.e., semantics and syntax of a computer language to achieve a particular computing task. For historical reasons, the majority of programming languages and programming paradigms are derived from English semantics and syntax, and implemented using ASCII character sets. A HCI model of interaction and communication between the programmer and the computer for programming tasks can occur at conceptual, cognitive, cultural, semantic and syntactic levels, as follows.

- $\quad$ Conceptual Level: The meaning of the task model should match the native user's world model and because this is where the concepts of programming, programming language features, relationships, and operations reside. At the conceptual level, the new technologies, complex concepts, unfamiliar metaphors, unnatural programming paradigms, lack of pedagogical concerns constitute a serious conceptual gap for the majority of native users. Native users make it more complicated because of their lack of knowledge and misunderstanding and misconceptions about computing tasks (Bayman \& Mayer, 1983; Boulay, 1989; Coombs et al., 1982; Mayer, 1981).

- Semantic Level: The ideas from the conceptual level are represented by means of a programming language placed in the context of a computing task. The abstract operations from the conceptual level are fully defined in terms of objects, functions and programming constructs of a programming language. At the semantic level, the semantics of programming do not match well with the natural semantics of native users' languages. In novice programming studies, it is observed in (Bonar \& Soloway, 1985; Ebrahimi, 1994; Dyck \& Mayer, 1985) that, the majority of semantic errors arise due to the semantic mismatch and novice users relaying on preconceived knowledge and translating the programming plans using their natural language semantics.

- Syntactic Level: At the syntactic level, the grammar of the programming language is defined, including the arrangement of valid programming elements and program construction. At the lexical level, the primitive elements such as tokens, programming 
vocabularies and literals of a programming language are used to write a program to achieve a computing task. The grammar at the syntax level is highly inflexible and the ASCII-based lexical tokens at the lexical level do not cover the majority of writing systems, complex scripts and diacritics.

- Cognitive Level: Cognition plays a vital role in computer programming. Each programming construct has its own cognitive load. Most programming languages use grammatical cues such that subject precedes the verb. These grammatical cues have diverse interpretations in other natural languages and cultures (e.g., Arabic, Hebrew, and Indian). These mismatching grammatical cues in programming languages introduce cognitive loads and have serious implications to programming constructs.

- Cultural Issues: Cultural factors, locale conventions and standards, bi-directional display of textual languages are not supported in the current programming languages and computing environments. These cultural factors help to motivate the users and enhance end user acceptability.

\section{EQUAL UNIVERSAL DESIGN AND DEVELOPMENT METHODOLOGY}

Visual programming systems, programming by demonstration, intelligent tutors and form-based programming may address some of the native users programming issues by providing native customised graphical programming environments. Visual languages for programming may help, but highlight only a few aspects of programming and manipulation of textual information structures. But, it is observed in (Gilmore \& Green, 1984; Green \& Petre, 1992) that the textual languages are more useful in learning the skills of programming such as comprehension, creation, documentation, modification and debugging and learning programming.

Universal Design or Design for All is a design methodology that recognises, respects, values and attempts to accommodate the broadest range of human abilities, skills, requirements and preferences in the design of all computer based products and environments. With about 6000 languages spread across some 200 countries, design of universally accessible textual information structures, programming languages and computing resources with diverse needs and preferences, requirements, a wide range of human capabilities, including native users with special needs is a difficult and a challenging task. EQUAL (Easy, Quick, Universal, Accessable Language) is a user-centred, universal design methodology that has been explicitly 
developed to support native users' requirements in the domain of textual languages (Patil et al., 2001a; Patil et al., 20001b). The EQUAL system provides a design and development environment that supports an universal usability engineering cycle with goals satisfying a broad range of the universal usability attributes such as availability, learnability, understandability, accessibility, and acceptability of textual programming languages and interactions.

The EQUAL universal usability engineering process and methodology consists of comprehensive set of guidelines on linguistic, cognitive, semantic and syntactic, cultural aspects of programming languages and computing resources. A basic guiding principle speak native users' language is exploited at language features, in compiler/interpreter components, and in editor and programming environment.

In general, there has been a conscious effort to make programming languages as natural as possible. It is observed that natural language-like constructs and commands are effective in the design of user interactions with the computer systems (Ledgard et al., 1980; Miller, 1981; Landauer et al., 1983; Biermann et al., 1983). Even recent studies (Pane et al., 2001) demonstrate the concept of "natural programming". Natural programming does not mean that native users should use their natural languages for programming, but the languages of programming and interactions should be as natural as possible and able to mentalize the computations and operations, learn quickly and use effectively the programming skills.

The empirical studies in (Carroll, 1978; Teasley, 1994) have demonstrated that meaningful variable names improved program comprehension and facilitate learning. Both formal and informal observational studies (Landauer et al., 1983; Rosenberg, 1982) repeatedly identified names, naming and structural contexts, and commands as an important practical problem The general interpretation of statement termination symbols, separators, programming cues, string constants (e.g., ';' is used for question mark in Greek, «string constant» in French, „string constant" in German) have diverse representations and interpretations in various languages and cultures. These notational representation and semantics of operators (e.g., relational, logical) are quite confusing and discouraging to many native users. A majority of native users misinterpret the meaning of relational operators and it is mainly due to lack of knowledge of Boolean algebra.

Cognitive processes and structures, program constructs play a vital role in problem solving, programming and comprehension (Hoc \& Nguyen, 1990; Rogalski \& Samurcay 1994). Control strategies and looping constructs are difficult concepts and demand high cognitive loads (Soloway et al., 1983). It may be observed that, just by providing equivalent controls 
and looping constructs in native programming language constructs may be insufficient and inadequate, and some time quite misleading and confusing from preconceived knowledge (Bonar \& Soloway, 1985). In IF-THEN statement and its variants, it is observed that (Pane et al., 2001) end-users use THEN for "sequencing" which contradicts the meaning of "consequently" in the formal languages. In REPEAT or WHILE control constructs, native users fail to understand the terminating or exit conditions. Such an understanding would lead to better programming languages whose syntactic structure more closely reflects internal semantic structures thereby easing the process of programming.

EQUAL universal design framework supports these notational flexibility and adaptations, and enhances easy comprehension and facilitates effective learning. Simple adaptations will reduce cognitive loads, avoid unnecessary frustrations and motivate native students to learn programming skills. The EQUAL design process may also be suitably transcribed into various native Braille codes. The universal design process consists of the following major steps.

- Collect Native User Requirements: The native user requirements, needs, and preferences, skill levels are collected and stored in the database as user profiles.

- $\quad$ Specify HCI Specifications: HCI experts can specify the human factors (e.g., linguistic, cultural, cognitive) and locale standards information, that play vital role in textual information structures, programming constructs and vernacular computing environments.

- Specify Language Design Constraints: The grammar of the EQUAL language may be expressed by regular expressions (RE) or extended BNF (EBNF) notations by the language designers. Each context-free grammar generates language, which is a set of strings of terminal symbols from the production rules. By changing the grammar, it is possible to incorporate different computing paradigms (e.g., declarative, procedural).

- Generate Language and Compiler Components: From the specifications generate (automatic or semiautomatic) the language and its compiler components such as scanners, lexical analysers and parsers. Special morphological data and language engineering skills are necessary to deal with complex writing systems and scripts.

- Evaluate Language and Compiler Components: After generating a language and its compiler components, the $\mathrm{HCI}$ experts, language designers and native users can test and evaluate the programming language and compiler components. 
We illustrate how linguistic, cultural, syntactic and semantic specifications and constrains are used in the EQUAL design space and are integrated into the EQUAL design and development process cycle. From a language designer's perspective, a syntax tree of grammar $G$ is an ordered label tree such that:

- the terminal nodes are labelled by terminal symbols;

- the non-terminal nodes are labelled by non-terminal symbols;

- each non-terminal node labelled by $\mathrm{N}$ has children, represented by

$\mathrm{X}_{1}, \mathrm{X}_{2}, . ., \mathrm{X}_{\mathrm{n}}$ such that $\mathrm{N}::=\mathrm{X}_{1}, \mathrm{X} 2, . ., \mathrm{X}_{\mathrm{n}}$ is a production rule.

A phrase grammar $G$ is a string of terminal symbols labelling the terminal nodes of a syntax tree. A sentence of grammar G generated is an S-phrase where $S$ is a start symbol. Since, the concrete syntax has no influence on the semantics of the language, this feature is exploited thoroughly to map various linguistic and cultural notations in a flexible manner. The guiding principle is to separate syntactic elements or vocabularies and semantic concepts and represent them in native users' language independent, culture neutral forms in the design space. The EQUAL design process is a multilevel, iterative process and modelled with a set of production rules. The production rules fire an action, when all the necessary universal usability criteria are satisfied. Any inconsistent or conflicting design decisions are reported to the designers. Any modifications and recommendations may be incorporated in the designevaluate-redesign cycle of EQUAL universal design methodology. The majority of programming statements (e.g., assignment, input, and output) has a generic format: $<$ verb $><$ operator $><$ variable $><$ terminator $>$. These generic formats have different representations and diverse interpretations in native languages and cultures. In localising these programming statements, the verb may be represented by one or more words and depends on number of variables, gender and polity. The terminating symbol may be represented by locale conventions and standards used in general communication.

In novice programming environments, it is observed (Eisenstadt, 1983; Witschital, 1994) user-friendly programming environment and visualisation of operations and computations helps in learning. The diagnostic and visualisation information may be used in feedback, tracers, debuggers or intelligent tutorials (Soloway et al., 1981; O'Shea \& Monk, 1981). The EQUAL programming environment provides this visual feedback and helps to acquire operational details of programming constructs.

Even simple literal translation or transliteration process of textual languages is difficult to cover all languages and cultures and may serve the following purposes:

- Helps in generating localised programming languages and computing environments for un-represented native users. (e.g., Pascal in Shona or Arabic). 
- Offers deeper insights into the universal usability issues of textual languages and helps in universal usability studies.

- Enables generation of equivalent representation in conventional programming languages, i.e. programming constructs in native languages and standard languages (e.g., Pascal in Arabic and English) and helps in scaffolding and learning.

\section{EQUAL SYSTEM ARCHITECTURE AND IMPLEMENTATION}

The majority of functional components are implemented using Java and exploit its internationalisation features and XML technologies. The EQUAL system supports Unicode standards that cover major scripts, symbols, and Unicode Braille patterns. The major functional components of the EQUAL system architecture are shown in Figure 1.

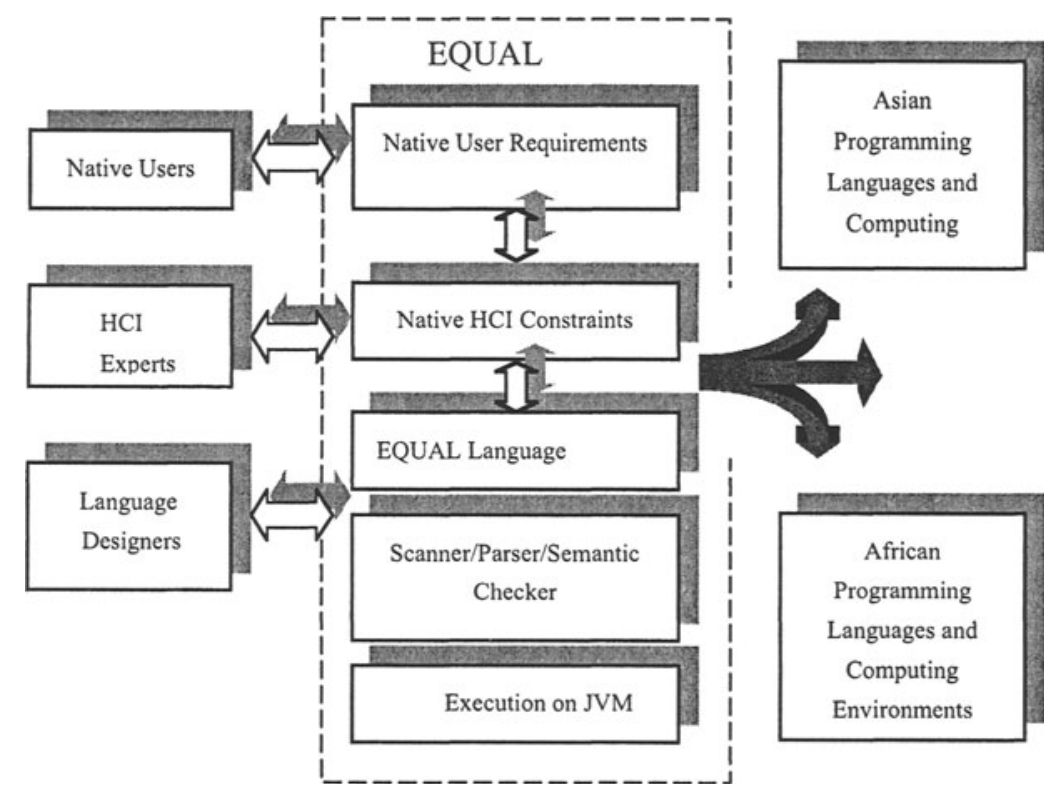

Figure 1. Architectural components of EQUAL design and development environment. 
The components consist of:

- EQUAL Grammar Specifier: Regular expressions or EBNF notations may express the grammar of the language by the language designers. Each context-free grammar generates language, which is a set of strings of terminal symbols. The concrete syntax is important to the implementers to check the syntactically well-formed programs. But the concrete syntax has no influence on the semantics of the program and used to represent the grammar in a flexible way. It may be observed that by changing the production rules, different programming paradigms may also be incorporated in the design process.

- EQUAL Database: The universal usability guidelines are stored in the relational database in the form of production rules. The database also includes the syntactic elements or vocabularies, and compiler components. A XML language is used to represent the metadata about the user profiles, cultural and linguistic factors, compiler components, and syntactic and semantic specifications. This metadata is used extensively at various stages of EQUAL development cycles of language design and generation of compilers and interpreters. The database may be extended and enriched by the HCI experts and language designers.

- Compiler/Interpreter Components: These components (e.g., lexical analyser, parser) are mainly concerned with the scanning or lexical analysis in which the source program written EQUAL is transferred into the streams of tokens and executed on a Java virtual machine.

\section{NATIVE USER COMPUTING ENVIRONMENT}

The user interfaces to systems for programming and computing environment can critically influence the development of programming skills. The EQUAL computing environment is similar to a conventional integrated programming environment consisting of multiple windows, GUI interface for programming, editor and language compiler/interpreter are localised to the computing requirements of native users. The native users can write EQUAL programs using Unicode compatible text editor and submit them for the compilation. The syntactic and semantic errors are displayed in the Error Window with necessary information to facilitate easy correction. In case of an error-free program, the interpreter/compiler will execute the program on Java Virtual Machine (JVM). The Input Window expects the inputs to the program and generates the outputs in the Output Window. Simple EQUAL 
programs are shown in Figure 2 supporting imperative style in Arabic or procedural i.e. PASCAL-like in Zimbabwean.

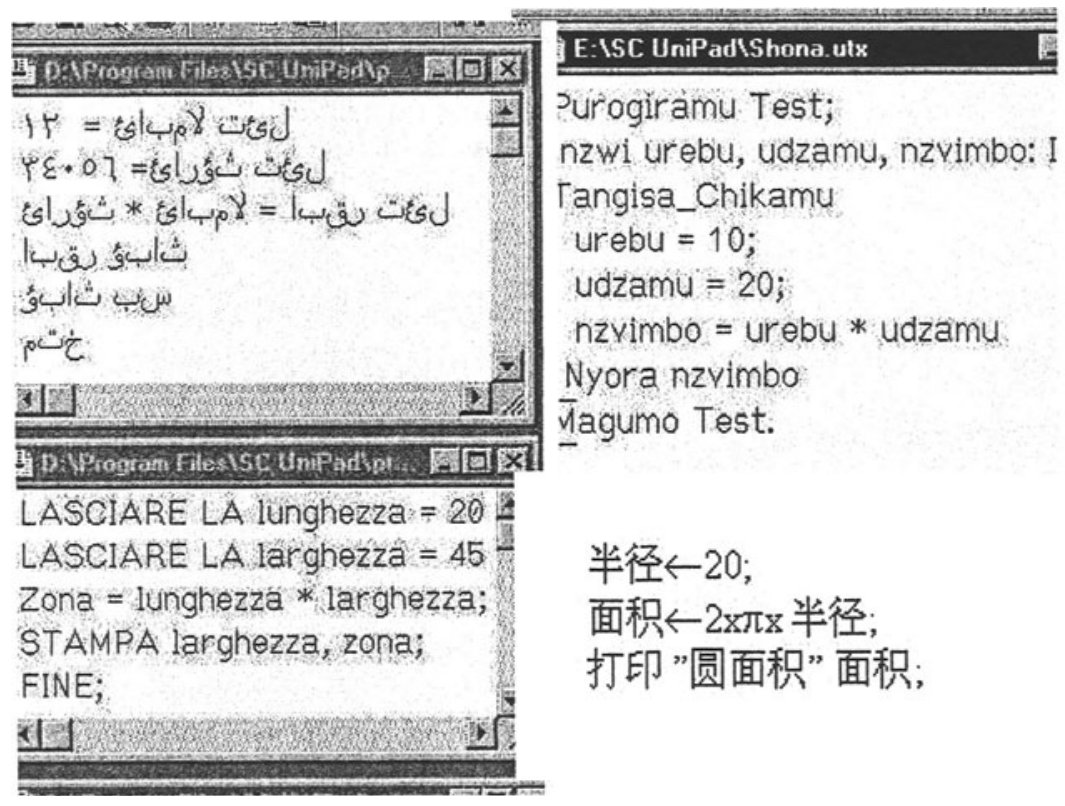

Figure 2. Cropped screen shots of EQUAL programs in Arabic, Zimbabwean (Shona) Italian and Chinese.

Online help and documentation are available in the Help Window. The error messages, dialogues, training and learning material are stored as resource bundles. The programming environment also generates the equivalent programs in English and native language and used for scaffolding.

The EQUAL language and computing environment uses Unicode for encoding to cover a majority of writing systems, cultural conventions, scientific and communication symbols. The majority of EQUAL language features and compiler/interpreter components are implemented using Java, and exploits its internationalisation and localisation features to support vernacular computing. These functional components may be deployed as standalone application or client-server model and helps in distribution and dissemination of learning resources for programming. 


\section{CONCLUSIONS AND FUTURE WORKS}

In this paper, we have discussed the universal usability issues of textual languages, novice programming languages and computing environments. We have discussed the universal design framework that can accommodate linguistic, cognitive, cultural, semantic and syntactic requirements of native programming languages and vernacular computing. Availability of native programming languages and computing resources is a pre-requisite attribute of universal usability and EQUAL fulfils availability of resources, by developing native textual languages and computing resources. We have shown the feasibility of the EQUAL language and its computing environments in a few representative nativised languages and computing environments. We believe that, the EQUAL language and EQUAL programming environment provides scaffolding mechanisms and offer unique opportunities to the native users to learn programming and computing skills. The EQUAL rapid prototyping environment helps in conducting universal usability studies in textual languages. Universal usability studies related to learnability, scalability, understandability, transfer of programming competence and critical evaluations are planned in the future work.

\section{REFERENCES}

Bayman, P. and Mayer, R.E. (1983). A Diagnosis of Beginning Programmers' Misconceptions of BASIC Programming Statements. Communications of the ACM, 26(9), 677-679.

Biermann, A.W., Ballard, W.B.W. and Sigmon, A.H. (1983). An Experimental Study of Natural Language Programming. International Journal of Man-Machine Studies, 18, 7187.

Bonar, J. and Soloway, E. (1985). Pre-Programming Knowledge: A Major Source of Misconceptions in Novice Programmers. Human-Computer Interaction, 1, 133-161.

Boulay, B. (1989). Some Difficulties of Learning to Program. In Studying the Novice Programmer, Soloway, E. and Sphrer, J.C (Eds.). New Jersey: Lawrence Erlbaum Associates.

Carroll, J.M. (1978). Names and Naming: An Interdisciplinary Review. IBM Research Report RC 7370, 1978.

Coombs, M.J., Gibson,R. and Alty, J.L. (1982). Learning a First Computer Language: Strategies for Making Sense. International Journalof Man-Machine Studies, 16, 449-486. 
Dyck, J.L, and Mayer, R.E. (1985). BASIC Versus Natural Language: Is There One Underlying Comprehension Process? Human Factors in Computing Systems - II, Proceedings of the CHI' 85 , San Francisco, USA,221-223.

Dyson, E. (1997). Education and Jobs in the Digitial World. Communications of the ACM, 40(2), 35-36.

Ebrahimi, A. (1994). Novice Programmer Errors: Language Constructs and Plan Composition. International Journal of Human-Computer Studies, 41, 457-480.

Eisenstadt, Marc. (1983). A User-friendly Software Environment for the Novice Programmer. Communication of the ACM, 26(12), 1058-1064.

Gilmore, D. J. and Green, T.R.G. (1984). The Comprehensibility of Programming Notations. HCI-Interact'84: Proceedings of the IFIP Conference Organised by Task Group on HCI, London, U.K, 461-464.

Green, T.R.G. and Petre, M. (1992). When Visual Programs are Harder to Read than Textual Programs. Human-Computer Interaction:Tasks and Organisation, Proceedings of ECCE-6 ( $6^{\text {th }}$ European Conference on Cognitive Ergonomics), van der Veer, G.C, Tauber, M.J., Bagnaroa, S. and Antavolts, M. (Eds.), Rome, Italy.

Hoc, J.M and Nguyen-Xuan.A (1990). Language Semantics, Mental Models and Analogy. In Psychology of Programming, Hoc, J.M, Green, T.R.G, Samurcay, R and Gilmore, D.J (Eds.). London:Academic Press.

Landauer, T.K., Glotti, K.M. and Hartwell, S. (1983). Natural Command Names and Initial Learning: A Study of Text Editing Terms. Communications of the ACM, 26, 495-503.

Ledgard, H., Whitehead, J.A., Singer, A. and Seymour, W. (1980). The Natural Language of Interactive Systems. Communications of the ACM, 23, 556-563.

Mayer, R.E. (1981). How Novices Learn Computer Programming. Computing Surveys, 13(1), 121-141.

Miller, L.A. (1981). Natural Language Programming: Styles, Strategies and Contrasts. IBM Journal, 20(2), 184-215.

O'Shea, Tim. and Monk, John. (1981). The Black Box Inside the Glass Box: Representing Computing Concepts to Novices. International Journal of Man-Machine Studies, 14, 237 249.

Pane, J.F, Ratanamahatana, C.A. and Myers, B.A (2001). Studying the Language and Structure in Non-programmers's Soultions to Programming Problems. International Journal of Human-Computer Studies, 54(2), 237-264.

Patil, Basawaraj., Maetzel, K. and Neuhold, E.J. (2001a). Design and Implemenation of Universal End-User Commands, Interfaces and Interactions. UAHCI, 2001, New Orleans, USA, 516-520.

Patil, Basawaraj., Maetzel, K. and Neuhold, E.J. (2001b). Native End-User Languages: A Design Framework. 13th Annual Workshop on Psychology of Programmng, Bournmouth, UK, 113-126.

Rogalski, J. and Samurcay, R. (1994). Acquisition of Programming Knowledge and Skills. In Psychology of Programming, Hoc, J.M.,Green, T.R.G., Samurcay, R. and Gilmore, D.J (Eds.). London: Academic Press. 
Rogers, P.L. (2000). Barriers to Adopting Emerging Technologies in Education. Journal of Educational Computing Research, 22(4), 455-472.

Rosenberg, J. (1982). Evaluating the Suggestiveness of Command Names. Behaviour and Information Technology, 1, 370-400.

Shneiderman, B. (2000). Universal Usability. Communications of $A C M, 43(5), 85-91$.

Soloway, E., Bonar, J., and Ehrlich, K. (1983). Cognitive Strategies and Looping Constructs:An Empirical Study. Communications of the ACM, 26(11), 853-860.

Soloway, E., Wolf, B., and Barth, P.(1981). MENO-II: An Intelligent Tutoring System for Novice Programmers. Proceedings of the Seventh International Joint Conference on Artificial Intellegence, Vancouver, 975-977.

Teasley, B.E. (1994). The Effects of Naming Style and Expertise on Program Comprehension. International Journal of Human-Computer Studies, 40, 757-770.

Witschital, Peter. (1994). TRAPS - An Intellgent Tutoring Environment for Novice Programmer. In Cognition and Computer Programming, Karl F. Wender, Franz Chmalhofer and Heinz-Dieter Böcker (Eds.). New Jersey: Ablex Publishing Corporation. 